\section{Hier steht eine Anzeige.}

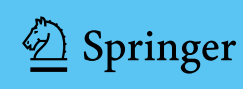

\title{
Bei Hautabszessen immer auch ein Antibiotikum?
}

\author{
Die Frage, ob sich bei Hautabszessen an Inzision und Drainage eine Antibiose \\ anschließen sollte, ist auch heute noch nicht ganz geklärt. Dies herauszufinden, \\ hat sich ein Forscherteam aus den USA zur Aufgabe gestellt.
}

$\mathrm{H}$ autärzte aus den USA konnten für eine prospektive placebokontrollierte Multicenterstudie 786 Teilnehmer gewinnen, darunter 281 Kinder. Um aufgenommen $\mathrm{zu}$ werden, mussten die $\mathrm{Pa}$ tienten einen einzelnen kutanen Abszess von maximal $5 \mathrm{~cm}$ im Durchmesser (bei Kindern bis acht Jahre maximal $4 \mathrm{~cm}$ ) haben. In allen Fällen wurde der Abszess zunächst per Inzision entleert, dann wurde drainiert. Aus dem Abszessinhalt ließen die Forscher Kulturen anfertigen.

Die Studie war dreiarmig angelegt: Im ersten Arm erhielten die Patienten dreimal täglich zwei 150-mg-Tabletten Clindamycin, im zweiten zweimal täglich zwei Tabletten mit $80 \mathrm{mg}$ Trimethoprim und $400 \mathrm{mg}$ Sulfamethoxazol (TMPSMX). Bei Kindern wurde die Ration altersentsprechend angepasst. Um die Verblindung zu gewährleisten, wurde der TMP-SMX-Gruppe als Mittagsration ein Placebomedikament gegeben. Die dritte Gruppe erhielt dreimal täglich Placebo.

Die Heilungsraten lagen gut eine Woche nach Beendigung der zehntägigen Therapie bei $83,1 \%$ in der ClindamycinGruppe, bei $81,7 \%$ in der TMP-SMXGruppe und bei 68,9\% in der Placebogruppe. Damit waren beide Verumstrategien dem Scheinpräparat signifikant überlegen.

In der Gesamtauswertung unterschieden sich die antibiotischen Regime nicht nennenswert in ihrer Erfolgsrate, wohl aber in Teilanalysen: So profitierten vor allem Kinder von der Clindamycin-Therapie deutlich mehr als von dem Kombipräparat, allerdings nur dann, wenn man ausschließlich die Patienten berücksichtigte, die die Studienmedikamente auch tatsächlich über die vorgeschriebene Dauer eingenommen hatten. Durchgehend adhärent waren 343 Teilnehmer.

Eine Kultur aus der Abszessflüssigkeit konnte in 781 Fällen angelegt werden. Dabei wurde in $67 \%$ Staphylococcus aureus isoliert, in knapp $50 \%$ der Gesamtteilnehmerzahl Methicillin-resistente Stämme (MRSA). Koagulase-negative
Staphylokokken fanden sich in $13 \%$ der Fälle; daneben gab es noch Streptokokken $(6,9 \%)$ und weitere Organismen. Auch bei den S.-aureus-infizierten Patienten waren nach abgeschlossener Therapie in den beiden Antibiotikagruppen signifikant mehr Patienten geheilt als in der Placebogruppe. Das Gleiche galt für Patienten mit MRSA.

Einen Monat wurde der Therapieerfolg überprüft. Zu diesem Zeitpunkt waren immer noch signifikant mehr Patienten aus der Clindamycin- und der TMP-SMX-Gruppe beschwerdefrei $(78,6 \%$ und $73,0 \%)$ als in der Placebogruppe $(62,6 \%)$. Von denen, die bei der ersten Kontrolluntersuchung geheilt waren, hatten $6,8 \%$ aus der Clindamycin- und $13,5 \%$ aus der TMP-SMXGruppe erneut eine Infektion entwickelt. In der Gruppe mit dem Kombipräparat waren es also signifikant mehr, die Rate lag ähnlich hoch wie unter Placebo (12,4\%).

Dafür gab es unter Clindamycin deutlich mehr Nebenwirkungen (21,9\% vs. $11,1 \%$ vs. $12,5 \%)$. Am häufigsten litten die Patienten unter leichtem Durchfall und Übelkeit, diese sistierten jedoch in der Regel von allein und blieben ohne weitere Folgen. In einem Fall entwickelte allerdings ein Patient unter TMP-SMX eine Überempfindlichkeitsreaktion mit Fieber, Ausschlag und Thrombozytopenie, die wahrscheinlich mit der Medikamenteneinnahme in Verbindung stand.

Fazit: : Kleinere Hautabszesse heilen wohl besser, wenn man zusätzlich zu Inzision und Drainage ein orales Antibiotikum verabreicht. Allerdings beschränke sich der Nutzen wohl auf Patienten mit S.-aureus-Infektionen, so die Autoren. Zudem weisen sie darauf hin, vor der Wahl von Clindamycin lokale Resistenzlagen zu berücksichtigen.

Dr. Elke Oberhofer

Daum RS et al. A Placebo-Controlled Trial of Antibiotics for Smaller Skin Abscesses. N Engl J Med 2017; 376: 2545-55 